\title{
Integrated Fragility Hip Fracture Program: A Model for High Quality Care
}

\author{
Jensa C Morris, MD*, Anne Moore, DNP¹, Joseph Kahan, MD², Marc Shapiro, MD4, \\ Jinlei Li, MD, PhD³, Brooke Spadaccino, RN¹, Michael Baumgaertner, MD², Mary I O'Connor, MD 1,2
}

${ }^{1}$ Center for Musculoskeletal Care, Yale School of Medicine and Yale-New Haven Hospital, New Haven, Connecticut; ${ }^{2}$ Department of Orthopedics and Rehabilitation, Yale School of Medicine, New Haven, Connecticut; ${ }^{3}$ Department of Anesthesia, Yale School of Medicine, New Haven, Connecticut; ${ }^{4}$ Department of Emergency Medicine, Massachusetts General Hospital, Boston, Massachusetts.

BACKGROUND: Hip fractures are a significant cause of morbidity and mortality among elderly patients. Coordinated multidisciplinary care is required to optimize medical outcomes.

OBJECTIVE: To determine the effect of the implementation of standardized, evidence-based protocols on clinical outcomes and mortality in patients with fragility hip fractures.

INTERVENTIONS: A multidisciplinary group was convened to define best practices in fragility hip fracture care and implement a fragility hip fracture clinical protocol at Yale-New Haven Hospital. Clinical outcomes in 2015, prior to program initiation, were compared with 2018, after the program was well established.

MAIN OUTCOMES AND MEASURES: Measured outcomes included 30-day mortality, blood transfusion utilization, adverse effects of drugs, venous thromboembolic complications, sepsis, myocardial infarction, mechanical surgical fixation complications during the index admission, length of stay, 30-day readmission, unexpected return to the operating room (OR) and time to the OR.

RESULTS: The implementation of the Integrated Fragility Hip Fracture Program was associated with significant reductions in 30-day mortality from $8.0 \%$ in 2015 to $2.8 \%$ in $2018(P=.001)$. Significant reductions were also seen in use of blood transfusions ( $46.6 \%$ to $28.1 \%$; $P<.001$ ), adverse effects of drugs (4.0\% to $0 \% ; P<.001)$, length of stay (5.12 to 4.47 days; $P=.004)$, unexpected return to the OR (5.1\% to $0 \% ; P<.001)$, and time to the OR $<24$ hours $(41.8 \%$ to $55.0 \%, P=.001)$.

CONCLUSIONS: An Integrated Fragility Hip Fracture Program using multidisciplinary care, physician and nursing engagement, evidence-based protocols, data tracking with feedback, and accountability can reduce mortality and improve clinical outcomes in patients with hip fractures. Journal of Hospital Medicine 2020;15:461-467. (c) 2020 Society of Hospital Medicine
$\mathrm{H}$ ip fractures are a significant cause of morbidity and mortality among elderly patients. Patients with fragility hip fractures often carry multiple comorbid diagnoses with a significant risk of perioperative complications. After hip fracture, 30-day mortality has been reported as $3.3 \%$ to $17.2 \%$ with one-year mortality as high as $50 \%$.

Multidisciplinary care, ${ }^{2-5}$ surgery within 24 hours $(h),^{6-12}$ use of regional peripheral nerve blocks, ${ }^{13-16}$ restrictive blood transfusion strategies, ${ }^{17,18}$ tranexamic acid (TXA) use, ${ }^{19}$ pharmacologic deep venous thrombosis (DVT) prophylaxis, ${ }^{20}$ surgical site infection prevention protocols, ${ }^{21}$ early mobilization, ${ }^{22}$ and nutritional optimization ${ }^{23-25}$ have been individually shown to improve outcomes in hip fracture patients.

Our program sought to define, standardize, and implement evidence-based best practices to improve clinical care and

*Corresponding Author: Jensa C. Morris, MD; Email: jensa.morris@ynhh.org Published online first February 19, 2020.

Find additional supporting information in the online version of this article.

Received: July 30, 2019; Revised: October 16, 2019;

Accepted: November 27, 2019

() 2020 Society of Hospital Medicine DOI 10.12788/jhm.3365 outcomes of patients with hip fractures. We convened a Center for Musculoskeletal Care (CMC) Hip Fracture Oversight Group that included surgeons and advanced practice providers from Orthopedics; physicians from Internal Medicine Hospitalist, Geriatrics, Emergency Medicine, and Anesthesia; and representatives from rehabilitation services, nursing, care management, pharmacy, and performance improvement. With clinical input from all involved services, we developed evidence-based protocols to standardize the care of patients with fragility hip fractures from the time of the patient's evaluation in the emergency room to discharge and outpatient rehabilitation. The program was operationalized in February 2016.

This project was considered by the Yale University institutional review board (IRB) to be a quality improvement and, therefore, exempted from IRB approval.

\section{MATERIALS AND METHODS}

Yale-New Haven Hospital is composed of two main campuses. The York Street Campus (YSC) is the Level 1 Trauma Center. The St. Raphael's Campus (SRC) houses the CMC nursing units for elective lower extremity arthroplasty and spine procedures. Prior to 2016, patients with hip fractures were cared for equally at both Yale-New Haven Hospital campuses. Patients were 
TABLE 1. Care Processes Included in the Integrated Fragility Hip Fracture Program

\begin{tabular}{|c|c|}
\hline Care Process & Description \\
\hline \multicolumn{2}{|l|}{ Preoperative } \\
\hline ED order set & $\begin{array}{l}\text { ED order set for suspected hip fracture includes ED protocols, staphylococcus PCR screening, and vitamin D assay to ensure early } \\
\text { availability of tests for inpatient team }\end{array}$ \\
\hline Standardized, magnification controlled, ED radiography & AP pelvis, hip AP and lateral films, and femur AP and lateral films to evaluate for hip fracture \\
\hline Hip fracture alert & Communication from ED staff to orthopedics, anesthesia, and internal medicine hospitalist of hip fracture patient in the ED \\
\hline Femoral nerve block & Single-shot femoral nerve block offered in the ED to all eligible patients \\
\hline Admission order set & Orthopedic hip fracture order set including all hip fracture protocols \\
\hline Medical comanagement & All patients admitted to on-call orthopedic surgeon service with medical attending comanaging from admission to discharge \\
\hline Surgical site infection prevention bundle & Staph PCR screening, nasal decontamination, chlorhexidine bath, and perioperative antibiotic algorithm \\
\hline Pain management protocol & Pain medications selected and dosed for the geriatric population and integrated into order sets \\
\hline Preoperative checklist & Review of all necessary preoperative steps \\
\hline Patient engagement & Verbal counseling and written brochures provided to patient and family; Patient engagement survey at discharge \\
\hline \multicolumn{2}{|l|}{ Intraoperative } \\
\hline Hip fracture on-call surgeon & Shared community and faculty orthopedic hip fracture call schedule \\
\hline Hip fracture OR block time & Dedicated hip fracture OR block assigned daily \\
\hline Tranexamic acid administration & Perioperative tranexamic acid protocol to reduce blood loss \\
\hline \multicolumn{2}{|l|}{ Postoperative } \\
\hline Postoperative order set & $\begin{array}{l}\text { Orthopedic hip fracture order set including all postoperative hip fracture protocols and standardized radiographic imaging } \\
\text { for quality assessment }\end{array}$ \\
\hline Standardized DVT prophylaxis & Sequential compression devices unless ambulating. Preoperative SC heparin and postoperative enoxaparin. \\
\hline Blood management protocol & Restrictive blood transfusion protocol and perioperative tranexamic acid use \\
\hline Nutritional optimization & Malnutrition screening, reduced NPO time, preoperative carbohydrate loading, and high protein nutritional supplementation \\
\hline Pneumonia prevention bundle & Interventions to reduce perioperative pneumonia: early mobilization, avoidance of PPIs, out of bed for meals, and daily teeth brushing \\
\hline Early mobilization & Out of bed before breakfast the morning after surgery \\
\hline
\end{tabular}

admitted to both medical and surgical services with no standardization of hip fracture care processes. Surgeons were assigned based on availability. Frequently, patients were added on to the operating room (OR) schedule and did not undergo surgery until off-hours and after a prolonged waiting period.

Medical comanagement of patients with fragility hip fractures at our institution predated the start of our CMC Integrated Fragility Hip Fracture Program (IFHFP). Comanagement was instituted in 2012 at YSC and in 2014 at SRC but without standardized protocols. The IFHFP began in February 2016 with the centralization of all patients with fragility hip fractures to the SRC at Yale-New Haven Hospital. Emergency medical services directed patients with suspected hip fractures to the designated campus. A dedicated hip fracture OR was allocated daily with a hip fracture surgeon assigned by a shared community and faculty surgeon call schedule. Patients were encouraged but not required to accept care from the on-call hip fracture surgical attending. Anesthesia was notified of the arrival of a patient with a hip fracture in the emergency department, and if the patient consented and qualified, a single-shot femoral nerve block was performed. Patients were screened for nasal staphylococcal colonization and treated with povidone-iodine nasal decolonization, chlorhexidine wash, and antibiotics determined by staphylococcal status and type of surgical procedure planned. Preoperative and postoperative order sets were implemented that dictated the care processes as outlined in Table 1. Surgeons determined the choice of operative intervention as per usual; this included internal fixation and partial or total hip replacement. Detailed medical and surgical protocols are included in Appendix A.

Since the initiation of the IFHFP on February 1, 2016, the program has continued to advance with our experience. We used 
TABLE 2. Demographic Data

\begin{tabular}{lcc}
\hline & Baseline Year 2015 & Intervention Year 2018 \\
\hline Total volume & 275 & 434 \\
\hline Average age [mean (SD)] & $82.0(11.0)$ & $80.9(11.4)$ \\
\hline Female & $210(76.4 \%)$ & $313(72.1 \%)$ \\
\hline Male & $65(23.6 \%)$ & $121(27.9 \%)$ \\
\hline Ethnicity & & $21(4.8 \%)$ \\
Black or African American & $14(5.1 \%)$ & $393(90.6 \%)$ \\
White or Caucasian & $253(92.0 \%)$ & $20(4.6 \%)$ \\
Other/unknown & $8(2.9 \%)$ & $218.8(5.5)$ \\
\hline Body mass index [mean (SD)] & $24.4(5.3)$ & .21 \\
\hline ASA score & & 52 \\
ASA 1 & $3(1.3 \%)$ & $5(1.7 \%)$ \\
ASA 2 & $54(23.3 \%)$ & $57(19.3 \%)$ \\
ASA 3 & $145(62.5 \%)$ & $209(70.9 \%)$ \\
ASA 4 & $30(12.9 \%)$ & $24(8.1 \%)$ \\
Missing & 43 & 139 \\
\hline
\end{tabular}

Abbreviations: ASA, American Society of Anesthesiologists; SD, standard deviation.

the year preceding the start of the program as our baseline year (January 1, 2015, through December 31, 2015). The following years, 2016 and 2017, were a transition time during which our protocols were implemented. The intervention year was defined as January 1, 2018, through December 31, 2018. The outcomes during the intervention year were compared with the baseline year. It is important to note that our program has been in continuous evolution, including during the intervention year, with protocols created and refined as we gain experience.

Outcomes include 30-day mortality, transfusions, adverse effects of drugs, venous thromboembolic complications, sepsis, myocardial infarction, mechanical surgical fixation complications, length of stay, 30-day readmission rate, unexpected return to the $O R$, and time to operative intervention. Definitions of the outcome variables are reviewed in Appendix B.

\section{RESULTS}

There were 275 consecutive patients with hip fractures admitted to SRC in the baseline year (January 1, 2015, to December $31,2015)$ and 434 patients with hip fractures admitted in the intervention year (January 1, 2018, to December 31, 2018) after consolidation of the program to the single Yale-New Haven Campus and implementation of standardized care processes. Patient demographic data including age, sex, ethnicity, body mass index, and American Society of Anesthesiologists physical status classification were evaluated for the baseline year and intervention year and reported in Table 2. There were no differences in the demographics of patients between baseline and intervention years.

From baseline year to intervention year, 30-day mortality, transfusion, adverse effects of drugs, length of stay, unexpected return to $O R$, and time to $O R$ were all significantly reduced. Mortality within 30 days decreased from $8.0 \%$ to $2.8 \%$. The results are displayed in Table 3. No significant difference was seen in the incidence of venous thromboembolism, sepsis, myocardial infarctions, readmission at 30 days, or mechanical surgical fixation complications.

The Figure shows the 30-day IFHFP mortality rate as reported on a monthly basis starting on January 1 of the baseline year, 2015, and continuing through December 31 of the intervention year, 2018. The process interventions are mapped according to the date of initiation. The median mortality rate (including all data from January 1, 2015, to December 31, 2018) is indicated by the dotted line. From May 2018 to December 2018, each monthly mortality rate was recorded below the four-year median, a visual demonstration of the statistical significance seen in our mortality reduction from $8.0 \%$ in the baseline year to $2.8 \%$ in the intervention year.

\section{DISCUSSION}

Patients with fragility hip fractures are a medically complex and vulnerable population. The goal of the CMC IFHFP was to standardize the care of these high-risk patients in an effort to reduce time to the $\mathrm{OR}$, perioperative medical complications, time spent in the hospital, and ultimately mortality.

The barriers to implementing coordinated, multidisciplinary care are significant. In our case, we had a fragmented care model with fragility hip fracture patients cared for at two campuses, on different nursing units, with both community and faculty surgeons providing operative care, and with no predesignated primary team. We structured our program for equal sharing of call between community and faculty surgeons. However, there was distrust among the physician groups: Primary care physicians were concerned that their referral lines with orthopedic surgical colleagues would be fractured by the new shared call. Surgeons doubted that patients would be distributed equally 
TABLE 3. Results

\begin{tabular}{|c|c|c|c|}
\hline & Baseline Year 2015 & Intervention Year 2018 & $P$ Value \\
\hline Total volume & 275 & 434 & \\
\hline 30-day mortality & $22(8.0 \%)$ & $12(2.8 \%)$ & .001 \\
\hline Transfusion & $128(46.6 \%)$ & $121(28.1 \%)$ & $<.001$ \\
\hline Adverse effect drugs & $11(4 \%)$ & $0(0.0 \%)$ & $<.001$ \\
\hline Venous thromoembolic events & $6(2.2 \%)$ & $5(1.2 \%)$ & .35 \\
\hline Sepsis & $3(1.1 \%)$ & $1(0.2 \%)$ & .30 \\
\hline Myocardial infarction & $3(1.1 \%)$ & $1(0.2 \%)$ & .30 \\
\hline Mechanical surgical fixation complications & $2(0.7 \%)$ & $0(0.0 \%)$ & .15 \\
\hline \multicolumn{4}{|l|}{ Length of stay, days } \\
\hline Mean (SD) & $5.12(3.31)$ & $4.47(2.12)$ & .004 \\
\hline Median (Range) & $4.0(1-31)$ & $4.0(1-19)$ & $<.001$ \\
\hline Readmission 30-day & $25(9.1 \%)$ & $54(12.5 \%)$ & .16 \\
\hline Unexpected return to $\mathrm{OR}$ & $14(5.1 \%)$ & $0(0.0 \%)$ & $<.001$ \\
\hline Time to OR (h) & & & .001 \\
\hline$<24 \mathrm{~h}$ & $115(41.8 \%)$ & $238(55.0 \%)$ & \\
\hline$>24 \mathrm{~h}$ and $<36 \mathrm{~h}$ & $93(33.8 \%)$ & $125(29.0 \%)$ & \\
\hline$>36 \mathrm{~h}$ & 67 (24.4\%) & $70(16.2 \%)$ & \\
\hline
\end{tabular}

among community and faculty practices. Hospitalists feared that comanagement would mean surgeons abdicating responsibility for care. Surgeons worried that routine medical involvement would delay surgery and prolong the length of stay with excessive testing. In order to achieve consensus, address concerns, and allay fears, we engaged the primary care and surgeon leadership for their support at the onset of the program and held monthly large group meetings and many smaller sessions to advance objectives. We meticulously tracked data and frequently reported out to the involved groups.

As it is well established that operative intervention on a hip fracture is best completed within $24 \mathrm{~h}$ to optimize a patient's clinical outcomes, critical interventions were the designation of a hip fracture OR starting midday and expectation that surgery be performed the day after admission for medically cleared patients. Surgeons were able to book elective cases or outpatient clinic time in the morning. The morning hours prior to surgery allowed time for any final medical optimization, preoperative nursing care, and family discussions. Most surgeries were then completed by the primary OR staff during standard operating hours. Patients were out of the postanesthesia care unit and settled back on the orthopedic nursing unit in the early evening without a prolonged period of nil per os, bed rest, or sleep interruption.

While our protocol expected surgery the day after admission for medically cleared patients, we used surgery within 24 $h$ as a simple metric to compare baseline with intervention outcomes. With our hip fracture OR block time beginning midday, the majority of our medically cleared hip fracture patients would receive surgical treatment within $24 \mathrm{~h}$ of admission. Our data show a significant improvement in timeliness of surgical intervention from $41.8 \%$ of patients to the OR within 24 h in 2015 to 55\% in 2018. In 2017, we conducted an interval four-month audit involving a detailed chart review of all patients for whom surgery was delayed beyond $24 \mathrm{~h}$ from hospital admission. Chart review identified anticoagulation as the primary reason for surgical delay. Of patients who were eligible for surgery (medically stabilized and not therapeutically anticoagulated), 90\% underwent surgery within $24 \mathrm{~h}$ during this four-month period in 2017. This compares to an overall rate of surgery within $24 \mathrm{~h}$ of $57 \%$ during the calendar year 2017. We did not perform a subgroup analysis of outcomes in patients with time to OR of 24-36 h. From this study, we are therefore unable to draw any conclusion regarding time to surgery and mortality.

Our dedicated OR hip fracture block time was changed from 7:30 AM to 12:30 PM during 2016 per surgeon request (Figure). Patients admitted within the 24-hour time period from 7 AM the day prior to 7 AM the day of the OR block time undergo surgery in the 12:30 PM time slot. Any patient admitted from 7 AM until $12: 30$ PM is not scheduled until the following day's OR block time and would hence have a surgical delay of $30 \mathrm{~h}$ or more. To better understand the impact of the later OR block time, we included the outcome variable of time to OR of greater 


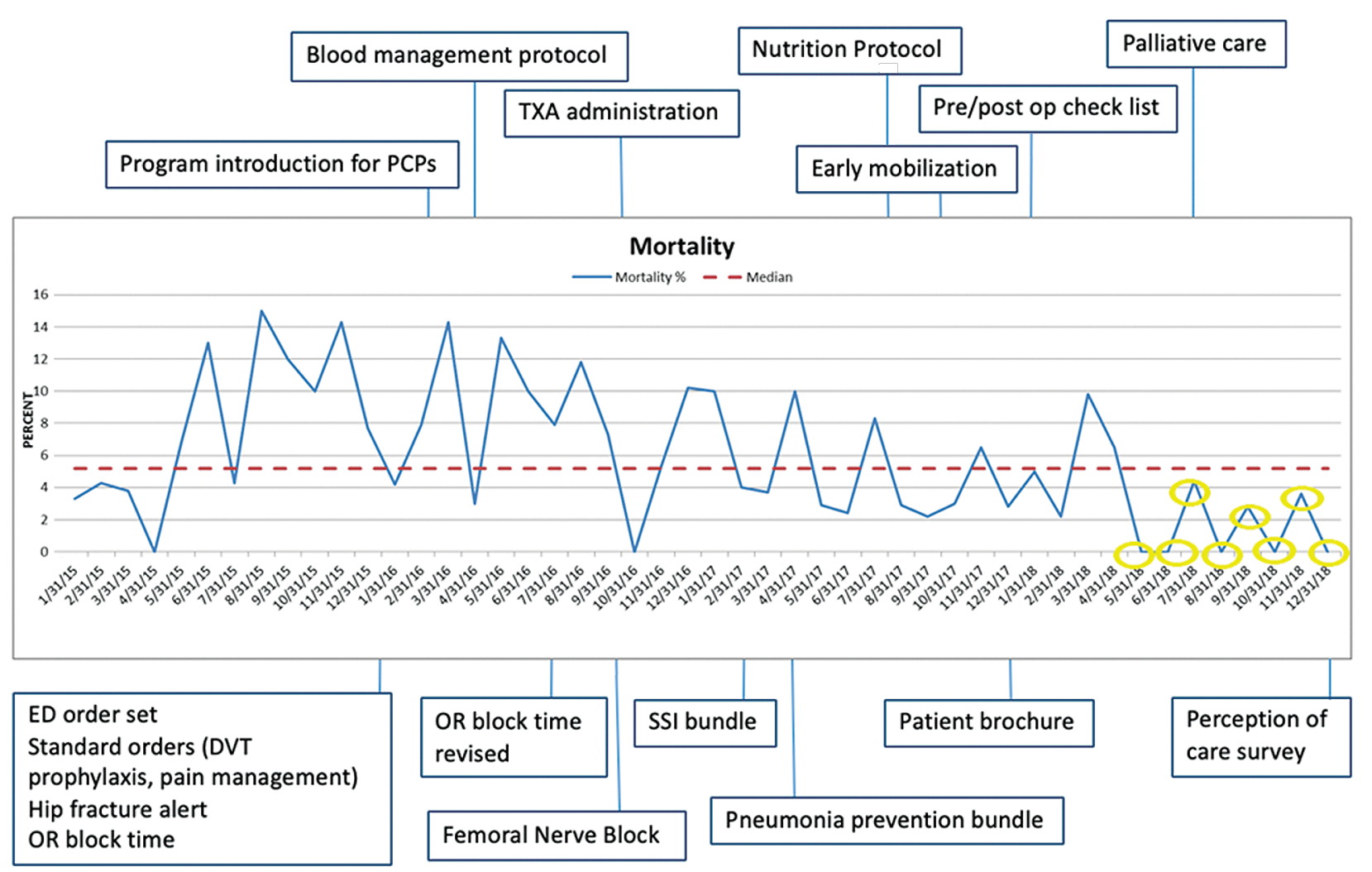

FIG. Timing of Implementation of Each Standardized Care Process Tracked Against 30-day Mortality Rate in the Fragility Hip Fracture Patient Population. Horizontal access is time, reported in monthly intervals from January, 2015 through December 2018. Vertical axis is 30-day mortality reported as a percentage of patients admitted with fragility hip fracture who died within 30 days of discharge. Red dashed line represents median mortality in the population from January 1, 2015 to December 31, 2018. In a quality improvement run chart, six consecutive data points below the median demonstrate statistically significant reduction in the outcome measured. The 8 data points from May 2018 to December 2018 all fall below the median 30-day mortality, demonstrating statistically significant mortality reduction.

NOTE: Abbreviations: DVT, deep vein thrombosis; ED, emergency department; OR, operating room; PCP, primary care doctor; SSI, surgical site infection; TXA, tranexamic acid.

than $24 \mathrm{~h}$ but less than or equal to $36 \mathrm{~h}$. We demonstrated a significant increase in the proportion of patients going to the OR in $24 \mathrm{~h}$ without an increase in patients waiting for 24 to 36 $h$ for their surgery.

Transfusion rate reduction from $46.6 \%$ to $28.1 \%$ was achieved primarily by the implementation and strict enforcement of a policy to avoid transfusing asymptomatic patients with hemoglobin $>7.0 \mathrm{~g} / \mathrm{dL}$. In addition, we recommended TXA using standard perioperative arthroplasty dosing of $1 \mathrm{~g}$ intravenously (IV) at the time of incision followed by $1 \mathrm{~g} \mathrm{IV} 3 \mathrm{~h}$ later in the postanaesthesia care unit. However, adherence to TXA recommendations was poor. A year-long audit (February 2017 to February 2018) demonstrated that only $29 \%$ of patients undergoing hip fracture surgery received the recommended TXA. After the conclusion of the study period of this review, we revised our TXA protocol to include an infusion at the time of admission and subsequent perioperative doses. The expanded TXA protocol (with clear exclusion criteria) has been "hard-wired" into our electronic perioperative order sets. We are tracking TXA compliance on a weekly basis. We anticipate that earlier TXA administration and improved compliance will further reduce transfusion rates.
We reduced the adverse effects of medications with two initiatives: First, dedicated hip fracture order sets with medications selected and dosed specifically for the geriatric population were launched at the onset of the IFHFP in 2016. Second, in coordination with our regional anesthesia team, patients who met criteria underwent a single-shot femoral nerve block upon diagnosis of the hip fracture. Patients reported up to $24 \mathrm{~h}$ of nonnarcotic pain relief with the femoral nerve block.

Prior to the introduction of the IFHFP, surgeons determined DVT prophylaxis based on their personal preference. Many of our surgeons were concerned that standardizing DVT prophylaxis to enoxaparin would increase the risk of surgical site bleeding, hematoma, infection, and reoperation. With data tracking and periodic reporting, we were able to reassure our surgeons: We demonstrated a reduction in the rate of patients unexpectedly requiring a return to the OR from $5.1 \%$ in 2015 to $0 \%$ in 2018.

We did not find a significant difference in mechanical complications due to surgical fixation during the index admission. Most mechanical complications do not present within the index admission and, therefore, would not be identified by this 
metric. Furthermore, in this phase of the program, we did not seek to change or standardize intraoperative surgical processes outside of surgical site infection prevention measures. Surgical technical quality and variation among surgeons is an area of ongoing evaluation within our program. We have begun a surgical quality review process with an expert review of postoperative radiography, beginning with fixation of nondisplaced femoral neck fractures, feedback to surgeons, and tracking of mechanical complications beyond the index admission. The surgical quality outcomes will be presented in a future manuscript.

Anticoagulation use is common in patients with hip fractures because of the high prevalence of comorbid conditions such as atrial fibrillation and venous thromboembolic disease. Direct oral anticoagulants (DOACs) are now commonly used in place of the vitamin $\mathrm{K}$ antagonist, warfarin. Our inability to efficiently reverse the DOACs compounded by surgeon unfamiliarity with these agents led to extreme caution in the timing of $O R$, with most patients delayed a full $48 \mathrm{~h}$ from the last dose of their prescribed DOAC. After recognizing the impact of anticoagulation on the timing of surgery, we convened a multidisciplinary group to determine rational guidelines for the timing of surgery in patients on chronic anticoagulation based on the bleeding risk of the planned operative procedure. These guidelines were instituted in December 2018, so their impact is not reflected in this review.

Our results showing a reduction in length of stay and mortality cannot be explained by any one intervention. We propose that the standardization of all processes and protocols, the establishment of clear expectations among all the medical and nursing personnel, and the shorter time spent waiting for surgery all contributed to the length of stay reduction. Likewise, the decrease in time to $O R$, reduction in time spent in the hospital, fewer transfusions, adverse effects of medication, and surgical complications requiring a return to the $O R$ have all likely contributed to the significant reduction in mortality. The efforts of the orthopedic nursing team certainly contributed: The CMC nurses led the efforts to standardize surgical site infection bundle care, reduce indwelling bladder catheter use, and together with physical therapy, mobilize patients out of bed for meals postoperatively. The strong focus on teamwork, data tracking, feedback and accountability, and the desire for continued improvement may have been the strongest drivers in this program's success.

Our results showed a nonsignificant increase in 30-day readmission from $9.1 \%$ to $12.5 \%$. One limitation of this study is that we did not track specific readmission diagnoses to better understand trends in diagnoses or indications for hospital readmission. Going forward, we are reviewing readmissions to better understand opportunities to improve our inpatient processes and transitions of care.

This an evolving project. We have expanded our use of TXA in an effort to further reduce transfusion rates. We have adjusted our protocols for patients admitted on DOACs and warfarin to allow more rapid surgical intervention. We have initiated a surgical quality review process in which surgical fixation is re- viewed with timely feedback to the operating surgeon. We are working closely with the skilled nursing facilities to extend our rehabilitation and nursing care protocols beyond the acute care setting. We are measuring patient engagement with a brief discharge survey specific to the CMC IFHFP. We continue to seek feedback from our referring primary care physicians to improve communication at times of care transition.

One of the limitations of a quality improvement project such as this one is the inability to identify the effect of each individual intervention. We can conclude that the totality of the multidisciplinary project reduced mortality in our hip fracture population, but we cannot report the relative effect of each process change. Another center seeking to replicate this success cannot determine from this research how to prioritize their resources to achieve a similar outcome.

How we care for the fragility hip fracture patient after hospital discharge is critical and unaddressed in this current study. A limitation of our current program is the lack of consistent postdischarge bone health management-which we are working to address. Also related to postdischarge management, we have partnered with a network of preferred skilled nursing facilities to standardize the care and decrease the length of stay. These data will be published separately.

We understand that our experience at the CMC is unique and specific to our care environment. This is a single site study and may not be generalizable to other centers. Nonetheless, the principles of multidisciplinary care, evidence-based protocol development, technological integration of protocols through order sets, and data tracking with feedback and accountability are the essential elements of our success that can be generalized to other institutions.

\section{CONCLUSIONS}

The CMC at Yale School of Medicine and Yale-New Haven Hospital IFHFP provides a model for implementing well-documented evidence-based interventions to standardize the care of patients with fragility hip fractures. The IFHFP yielded reduced mortality, length of stay, blood transfusion utilization, adverse effects of medications, unexpected return to the OR, and time to the OR.

\section{Acknowledgments}

The authors thank the work of the Center for Musculoskeletal Care Hip Fracture Oversight Group, program surgeons, and community primary care leaders: Olukemi Akande, MD, Mark Altman, MD, Diren Arsoy, MD, John Aversa, MD, Michael Connair, MD, Leo Cooney, MD, Kenneth Donohue, MD, David Gibson, MD, Gail Haesche, RN, MS, ACM-RN, Carol Just, MSN, NEA-BC, RN, Patricia Kenyon, RN, ACM, Francis Lee MD, Michael Leslie, MD, Michael Lucchini, MD, Christopher Lynch, MD, Rowland Mayor, MD, Tara Messina, PT, Lorraine Novella, RN, Paul Oliver, PA-C, Vivek Parwani, MD, Joseph Quaranta, MD, Lee Rubin, MD, Derek Shia, MD, Jeff Sumner, MD, John Tarutis, Arya Varthi, MD, Anuruddha Walaliyadda, MD, Daniel Wiznia, MD, Shirvinda Wijesekera, MD, Joseph Wu, MD, Brad Yoo, MD, and James Yue, MD.

Disclosures: Dr O'Connor reports personal fees from ZimmerBiomet, Inc., outside the submitted work. All other authors have nothing to disclose.

Funding: All IFHFP quality interventions were funded by existing $\mathrm{CMC}$ and YNHH budgets. 


\section{References}

1. Abrahamsen B, van Staa T, Ariely M, Olson M, Cooper C. Excess mortality following hip fracture: a systematic epidemiologic review. Osteoporos Int 2009;20(10):1633-1650. https://doi.org/10.1007/s00198-009-0920-3

2. DellaRocca GJ, Moylan KC, Crist BD, Volgas DA, Stannard JP, Mehr DR. Comanagement of geriatric patients with hip fractures: a retrospective, controlled cohort study. Geriatr Orthop Surg Rehabil. 2013;4(1):10-15. https:// doi.org/10.1177/2151458513495238

3. Wang Y, Tang J, Zhou F, Yang L, Wu J. Comprehensive geriatric care reduces acute perioperative delirium in elderly patients with hip fractures: a meta-analysis. Medicine. 2017; 96(26): e7361. https://doi.org/10.1097/ MD.0000000000007361

4. Liu VX, Rosas E, Hwang J, et al. Enhanced recovery after surgery program implementation in 2 surgical populations in an integrated health care delivery system. JAMA Surg. 2017;152(7):e171032. https://doi.org/10.1001/jamasurg.2017.1032

5. Taraldsen $K$, Sletvold $O$, Thingstad $P$, et al. Physical behavior and function early after hip fracture surgery in patients receiving geriatric care or orthopedic care-a randomized controlled trial. J Gerontol A Biol Sci Med Sci. 2014;69(3):338-345. https://doi.org/10.1093/gerona/glt097

6. Grimes JP, Gregory PM, Noveck H, Butler MS, Carson Jl. The effects of timeto-surgery on mortality and morbidity in patients following hip fracture. Am J Med. 2002;112(9):702-709. https://doi.org/10.1016/s0002-9343(02)01119-1

7. Hamlet WP, Lieberman JR, Freedman EL, Dorey FJ, Fletcher A, Johnson EE. Influence of health status and the timing of surgery on mortality in hip fracture patients. Am J Orthop. 1997;26(9):621-627.

8. Hoenig H, Rubenstein LV, Sloane R, Honer R, Kahn K. What is the role of timing in the surgical and rehabilitative care of community-dwelling older persons with acute hip fracture? Arch Intern Med. 1997;157(5):513-520.

9. Orosz GM, Magaziner J, Hannan El, et al. Association of timing of surgery for hip fracture and patient outcomes. JAMA. 2004;291(14):1738-1743. https:// doi.org/10.1001/jama.291.14.1738

10. Gdalevich M, Cohen D, Yosef D, Tauber C. Morbidity and mortality after hip fracture: the impact of operative delay. Arch Orthop Trauma Surg 2004:124(5):334-340. https://doi.org/10.1007/s00402-004-0662-9

11. Doruk H, Mas MR, Yidiz C, Sonmez A, Kýrdemir V. The effect of the timing of hip fracture surgery on the activity of daily living and mortality in elderly. Arch Gerontol Geriatr. 2004;39(2):179-185. https://doi.org/10.1016/j.archger.2004.03.004

12. Uzoigwe CE, Burnand HG, Cheesman CL, Aghedo DO, Faizi M, Middleton RG. Early and ultra-early surgery in hip fracture patients improves survival. Injury. 2013;44(6):726-729. https://doi.org/10.1016/j.injury.2012.08.025

13. Guay J, Parker MJ, Griffiths R, Kopp SL. Peripheral nerve blocks for hip fractures. Cochrane Database Syst Rev. 2017;5: CD001159. https://doi. org/10.1002/14651858.CD001159.pub2
14. Morrison RS, Dickman E, Hwang $U$, et al. Regional nerve blocks improve pain and functional outcomes in hip fracture: a randomized controlled trial. J Am Geriatr Soc. 2016;64(12):2433-2439. https://doi.org/10.1111/jgs.14386

15. Beaudoin $\mathrm{FL}$, Haran JP, Liebmann $\mathrm{O}$. A comparison of ultrasound-guided three-in-one femoral nerve block versus parenteral opioids alone for analgesia in emergency deparment patients with hip fractures: a randomized controlled trial. Acad Emerg Med. 2013;20(6):584-591. https://doi.org/10.1111/ acem. 12154

16. Dickman E, Pushkar I, Likourezos A, et al. Ultrasound-guided nerve blocks for intracapsular and extracapsular hip fractures. Am J Emerg Med. 2016;34(3):586-589. https://doi.org/10.1016/j.ajem.2015.12.016

17. Carson JL, Terrin MI, Noveck H, et al. Liberal or restrictive transfusion in highrisk patients after hip surgery. N Engl J Med. 2011;365(26):2453-2462. https:// doi.org/10.1056/NEJMoa1012452

18. Garcia-Alvarez F, Al-Ghanem R, García-Alvarez I, López-Baisson A, Bernal M. Risk factors for postoperative infections in patients with hip fracture treated by means of Thompson arthoplasty. Arch Gerontol Geriatr. 2010; 50(1):51-55. https://doi.org/10.1016/j.archger.2009.01.009

19. Farrow LS, Smith TO, Ashcroft GP, Myint PK. A systematic review of tranexamic acid in hip fracture surgery. Br J Clin Pharmacol. 2016;82(6):1458-1470. https://doi.org/10.1111/bcp.13079

20. Falck-Ytter Y, Francis CW, Johanson NA, et al. Prevention of VTE in orthopedic surgery patients: Antithrombotic Therapy and Prevention of Thrombosis, 9th ed: American College of Chest Physicians Evidence Based Clinical Practice Guidelines. Chest. 2012;141(2 Suppl):e278S-e325S. https://doi. org/10.1378/chest.11-2404

21. Gillespie WJ, Walenkamp G. Antibiotic prophylaxis for surgery for proximal femoral and other closed long bone fractures. Cochrane Database Syst Rev. 2010;(3):CD000244. https://doi.org/10.1002/14651858.CD000244.pub2

22. Kamel HK, labal MA, Mogallapu R, Maas D, Hoffmann RG. Time to ambulation after hip fracture surgery: relation to hospitalization outcomes. J Gerontol A Biol Sci Med Sci. 2003;58(11):1042-1045. https://doi.org/10.1093/ gerona/58.11.m1042

23. Foster MR, Heppenstall RB, Friedenberg ZB, Hozack WJ. A prospective assessment of nutritional status and complications in patients with fractures of the hip. J Orthop Trauma. 1990;4(1):49-57. https://doi.org/10.1097/00005131199003000-00009

24. Bell JJ, Pulle RC, Crouch AM, Kuys SS, Ferrier RL, Whitehouse SL. Impact of malnutrition on 12-month mortality following acute hip fracture. ANZ J Surg. 2016;86(3):157-161. https://doi.org/10.1111/ans.13429

25. Avenell A, Handoll HH. Nutritional supplementation for hip fracture aftercare in older people. Cochrane Database Syst Rev. 2010;(1):CD001880. https:// doi.org/10.1002/14651858.CD001880.pub5 